\title{
Spatial processing laterality and spatial visualization ability: Relations to sex and familial sinistrality variables
}

\author{
MICHAEL F. MARINO \\ Northport VA Hospital, Northport, New York \\ and \\ WALTER F. MCKEEVER \\ Northern Arizona University, Flagstaff, Arizona
}

\begin{abstract}
Seventy-four right-handed college students were administered the Clockface Reading Latency Task (CRLT) and the Stafford Identical Blocks Test (SIBT). The lateralized tachistoscopic CRLT yielded a significant left visual field superiority, supporting previous research that had suggested it was a sensitive test of right hemisphere superiority for spatial processing. On the SIBT, males scored significantly higher than females, and there was a sex $\times$ familial sinistrality (FS) interaction, like that previously reported by McKeever, Seitz, Hoff, Marino, and Diehl (1983). FS also influenced visual field asymmetries on the CRLT; subjects with left-handedness in their families showed smaller asymmetries. No correlation between spatial laterality and spatial ability was found.
\end{abstract}

McKeever and Hoff (1982) and Seitz and McKeever (1984) found a sex $\times$ familial sinistrality (FS) interaction on the Object Naming Latency Task (ONLT), a task that assesses language lateralization. In right-handed subjects, the sex $\times$ FS interaction indicated that females who lacked familial sinistrality (FS-) and males who had positive familial sinistrality (FS+) showed significantly smaller right visual field (RVF) superiorities on the language lateralization task than did FS+ females and FSmales. Thus, the data suggest that the former groups are less strictly left hemisphere dominant for language functions than are the latter groups. McKeever, Seitz, Hoff, Marino, and Diehl (1983) also found a sex $\times$ FS interaction in spatial visualization ability as measured by the Stafford Identical Blocks Test (SIBT; Stafford, 1961). On the SIBT, the FS - females and FS+ males scored higher than the FS+ females and FS- males.

One possible explanation for the sex $\times$ FS interaction in the ONLT is that the outcome was influenced by the spatial ability levels of the subjects. Since there is obviously a spatial component to rapid naming of object pictures, it might be that subjects who possess superior spatial ability are more right hemisphere dominant for that component. Indeed, Levy (1974) postulated that strong lateralizations of language and spatial abilities to the left and right hemispheres, respectively, are related to higher

This study was conducted at Bowling Green State University by the first author, under the direction of the second author, in partial fulfillment of the requirements for the Master of Arts degree. Address reprint requests to W. F. McKeever, Department of Psychology, Northern Arizona University, P.O. Box 15106, Flagstaff, AZ 86011. levels of verbal and spatial skills. Therefore, when object pictures are presented to the left visual half-field (VHF) on the ONLT, the right hemispheres of the subjects having high spatial ability might excel the left in speed of recognition, thereby diminishing the RVF superiority for speed of object picture naming. Because the FS - female and FS+ male subjects have greater spatial ability than the FS+ female and FS- male subjects, as assessed by the SIBT, greater right hemisphere recognition speed by the FS- females and FS+ males should reduce their RVF superiorities on the ONLT relative to those of the FS+ females and FS- males.

To test this possibility, one needs a more direct assessment of hemispheric dominance for spatial processing. Although various tasks designed to measure a right hemisphere dominance for spatial processing have been investigated, they have often failed to reveal LVF superiorities (see McKeever, 1986). A task that appears to be promising for assessing right hemisphere spatial processing superiorty is the Clockface Reading Latency Task (CRLT), originated by Berlucchi, Brizzolara, Marzi, Rizzolatti, and Umilta (1979). Berlucchi et al. found a clear LVF superiority for clockface reading, even though the dependent measure was the latency of vocal report of the time displayed. Apparently, the processing requirements of the task, such as judging the relative size and orientation of the two hands of the clock, are sufficiently right hemisphere-dependent to ensure LVF superiority despite the vocal nature of the final response. In the present experiment, we employed a modified version of the clockface reading task of Berlucchi et al. If the same pattern of sex $\times$ FS interaction as seen in the ONLT and the SIBT were demonstrated for spatial processing laterality, then 
it would seem reasonable to conclude that spatial processing laterality provides the basis for the interaction on the ONLT and SIBT.

\section{METHOD}

\begin{abstract}
Subjects
The subjects were 40 male and 34 female undergraduates. An additional 26 subjects (18 males and 8 females) were originally tested, but their error rates exceeded a predetermined criterion for inclusion (less than $10 \%$ error rate) in the study. All subjects were right-handed for writing, were under the age of 25 , and had normal or corrected-to-normal visual acuity.
\end{abstract}

\section{Apparatus}

A Gerbrands tachistoscope (Model 3B), equipped with an automatic card changer, was employed for stimulus presentation. Vocal reaction times (report of the time displayed on the clockfaces) were recorded from a Hunter Timer, in milliseconds. Luminance of the fixation and stimulus fields was $3.0 \mathrm{fL}$. Exposure time was $150 \mathrm{msec}$, with a 7-sec ITI.

\section{Clockface Reading Latency Task}

The original CRLT as employed by Berlucchi et al. (1979) was, in our judgment, too difficult to provide a valid reaction time task. The error rates were so high that for their latency analyses, they had to retain all subjects whose error rates were less than $50 \%$. Consequently, for our task, we employed only eight different times $(1: 25,2: 55,4: 10$, $5: 40,7: 50,8: 05,10: 35$, and 11:20). Among the guidelines for selecting these times were the following: neither hand could point to the 3 , 6,9 , or 12 positions on the clockface; the hour hand could be positioned only once between the same two marks on the dial; any combination of the hour and minute hands that would place both hands in the same quadrant of the clockface was not allowed; hour and minute hand combinations in which the two hands were on the same half of the clockface were to occur on one-half of the stimuli, with the constraint that the angles formed by the hands be equivalent for the two halves of the clockface; and since the hour and minute hands were located twice within each quadrant of the clockface, it was required that the hour and minute hands from each quadrant be paired with a complementary hour and minute hand combination on the opposite side of the clockface. Stimulus cards that displayed the chosen times were prepared. Each stimulus card depicted a circular clockface graduated into the usual $125-\mathrm{min}$ divisions, but these divisions were not numbered. Clockface diameter subtended $2.5^{\circ}$ of visual angle, with the nearest point to fixation corresponding to $1^{\circ}$ of visual angle. The hour and minute hands subtended $.75^{\circ}$ and $1^{\circ}$ of visual angle, respectively.

\section{Procedure}

Prior to the tachistoscopic trials, the subjects viewed replicates of the eight stimulus cards for two 40 -sec periods, and, following each period, they were asked to recall the times. Errors and omissions were brought to their attention. This procedure was designed to facilitate recognition, to teach the appropriate labels, and to reduce errors.

The subjects were instructed that on experimental trials, a tone (occurring $1.5 \mathrm{sec}$ prior to stimulus exposure) would signal presentation of a clockface to one side or the other side of the fixation dot, and that they were to read aloud the time presented as quickly and accurately as possible. They were instructed to report the hour first, and then the number of minutes (i.e., "'one twenty-five,"' seven fifty," etc.). In addition, subjects were told that several of the presented stimuli would consist of only a single digit located directly at the focus point. They were told that they must accurately report this digit any time it appeared, although their response to it would not be timed. Such number-only cards occurred randomly, twice during each block of 32 experimental trials, and were incorporated to help ensure that the subjects respected the instruction to fixate the fixation point.

Six blocks of 32 clockface stimuli were presented, with each clockface appearing twice in each VHF. The first two trial blocks were considered practice trials. Stimulus order was quasirandom, under the constraints that no more than three consecutive stimuli be in the same VHF and that consecutive trials not display the same times. The predetermined order of stimulus presentation was reversed between successive blocks. After each block, the subjects were given a brief rest of 1 to $2 \mathrm{~min}$. During this time they were told their fastest and slowest reaction times (RTs) to the previous block (never whether these occurred to particular half-field or stimulus trials), and were urged to try to attain still faster times during the up-coming block.

Throughout the experiment the subjects were corrected immediately following trials on which errors had occurred. Furthermore, each subject was read the correct times following the second block of trials, again to help reduce the potential problem of excessive error rates. Trials in which errors occurred were considered equivalent to the particular subject's longest RT. The basic dependent measures were median RTs to the LVF and RVF clockface presentations, and the median RTs were based on the 64 LVF and 64 RVF trials of the final four blocks.

The SIBT was administered upon completion of the CRLT, with a 15-min time limit. Finally, the subjects reported the incidence of sinistrality in their families. Two different definitions of FS were employed in analyzing the data. The first, traditional FS, considered subjects with at least one first-degree left-handed (for writing) relative or two seconddegree left-handed relatives as FS+. A second definition, termed strict $F S$, considered only those with first-degree left-handed relatives as FS+ and only those having absolutely no first- or second-degree left-handed relatives as FS-.

\section{RESULTS}

Mean median VHF latencies and LVF-RVF differences of the various subject groups and of all subjects are shown in Table 1. The FS definition employed in determining the data of Table 1 was the traditional FS definition employed in our laboratory. Mean scores on the SIBT are also shown. A three-way (sex $\times$ FS $\times$ fields) analysis of variance revealed a significant main effect of visual fields $[F(1,70)=15.64, p<.001]$. Thus, the CRLT was processed more efficiently by the right than by the left hemisphere. The main and interactive effects of the sex and FS factors were nonsignificant, although, as can be inferred from Table 1, there was a tendency for larger LVF superiorities in the FS- subjects $(p<.172)$. No other effects were significant. On the SIBT, males scored significantly higher than females, and there was a significant sex $\times$ FS interaction, like that reported by McKeever et al. (1983). The correlation of VHF asymmetries and SIBT scores (not shown in the table) was nonsignificant $(r=.04)$.

Table 2 provides data on the CRLT and SIBT scores for 59 subjects whose FS status was defined by the strict FS criterion. By excluding the 8 subjects whose previous FS+ status was based on left-handedness of second-degree

Table 1

Mean Reaction Times for LVF, RVF, and LVF-RVF Differences and SIBT Scores of Traditional FS Subject Groups

\begin{tabular}{lrrrr}
\hline \multicolumn{1}{c}{ Group } & \multicolumn{1}{c}{ LVF } & \multicolumn{1}{c}{ RVF } & DIFF & SIBT \\
\hline FS- Females & 997.6 & 1028.9 & -31.3 & 20.5 \\
FS+ Females & 1039.2 & 1059.4 & -20.2 & 14.7 \\
FS- Males & 926.4 & 959.5 & -33.1 & 21.1 \\
FS+ Males & 1017.4 & 1028.5 & -11.1 & 23.9 \\
All Subjects & 988.4 & 1013.4 & -25.0 & 20.1 \\
\hline
\end{tabular}

Note-LVF = left visual field. RVF = right visual field. DIFF = LVF-RVF difference. SIBT $=$ Stafford Identical Blocks Test. FS = familial sinistrality. 
Table 2

Mean Reaction Times for LVF, RVF, and LVF-RVF Differences and SIBT Scores of Strict FS Subject Groups

\begin{tabular}{lrrrr}
\hline \multicolumn{1}{c}{ Group } & \multicolumn{1}{c}{ LVF } & \multicolumn{1}{c}{ RVF } & \multicolumn{1}{c}{ DIFF } & SIBT \\
\hline FS- Females & 997.9 & 1012.8 & -30.7 & 20.0 \\
FS+ Females & 1052.8 & 1054.9 & -02.1 & 17.5 \\
FS- Males & 923.6 & 961.8 & -38.2 & 20.8 \\
FS+ Males & 1026.6 & 1032.1 & -05.5 & 23.7 \\
All Subjects & 991.0 & 1012.8 & -21.8 & 20.7 \\
\hline
\end{tabular}

Note-LVF $=$ left visual field. RVF $=$ right visual field. DIFF = LVF-RVF difference. SIBT $=$ Stafford Identical Blocks Test. FS $=$ familial sinistrality.

relatives and the 7 subjects whose previous FS- status ignored the existence of a second-degree left-handed relative, the sample size was reduced, but the exclusivity of the FS groups was increased. Three-way (sex $\times$ FS $\times$ fields) ANOVAs revealed a significant main effect of fields $[F(1,55)=7.48, p<.01]$. The interaction of FS and fields was also significant $[F(1,55)=4.83, p<.05]$. No other effects were significant. The results for the SIBT were similar to those found for the larger sample, although the sex $\times$ FS interaction was short of significance in the smaller sample $[F(1,55)=2.72, p=.10]$. Males scored significantly higher than females $[F(1,55)=4.50$, $p<.05$ ], as in the larger sample. The correlation of VHF asymmetries and SIBT scores was again nonsignificant $(r=-.01)$.

\section{DISCUSSION}

The results showed that a LVF superiority obtained on the CRLT. Thus, we infer a right hemisphere superiority for the processing required by the task. The data also show that FS influences the outcome, particularly when defined according to the strict FS definition. Indeed, when this definition was employed, the LVF superiority was due almost entirely to the FS- subjects. The data provide evidence, as reported by McKeever et al. (1983), that males score significantly higher than females on the SIBT and that this sex effect is influenced by the FS factor as well. When the traditional FS definition employed in our previous studies with the SIBT was used, the pattern of superior performance by FS - females and FS+ males was replicated. The hypothesis that these superior spatial ability groups would be more lateralized on a test of hemispheric specialization for spatial processing, however, was not supported in the case of FS + males. Thus, the FS $\times$ sex interaction obtained for language laterality on the ONLT in earlier studies cannot be ascribed to spatial ability influence. Finally, contrary to the general hypothesis (Levy, 1974) that spatial laterality and spatial ability are related, the results suggest that these factors are independent.

\section{REFERENCES}

Berlucchi, G., Brizzolara, D., Marzi, C. A., Rizzolatti, G., \& Umilta, C. (1979). The role of stimulus discriminability and verbal codability in hemispheric specialization for visuospatial tasks. Neuropsychologia, 17, 195-202.

LEVY, J. (1974). Psychological implications of bilateral asymmetry. In S. Diamond \& J. G. Beaumont (Eds.), Hemisphere function in the human brain. London: Elek.

MCKeEVER, W. F. (1986). Tachistoscopic methods in neuropsychology. In H. J. Hannay (Ed.), Experimental techniques in human neuropsychology. New York: Oxford University Press.

McKeever, W. F., \& Hoff, A. L. (1982). Familial sinistrality, sex, and laterality differences in naming and lexical decision latencies of right-handers. Brain \& Language, 17, 225-239.

McKeever, W. F., Seitz, K. S., Hoff, A. L., Marino, M. F., \& DieHL, J. A. (1983). Interacting sex and familial sinistrality characteristics influence both language lateralization and spatial ability in right handers. Neuropsychologia, 21, 661-668.

SeITZ, K. S., \& McKeEver, W. F. (1984). Unilateral versus bilateral presentation methods in the reaction time paradigm. Brain \& Cognition, 3, 413-425.

STAFFord, R. (1961). Sex differences in spatial visualization as evidence for sex-linked inheritance. Perceptual \& Motor Skills, 13, 428.

(Manuscript received July 1, 1988.) 